\title{
Valores Éticos en la Formación Universitaria de las Áreas de Ciencias Naturales e Ingeniería y Tecnología, en el Contexto de la Sociedad del Conocimiento
}

\author{
Cecilia Osuna ${ }^{(1)}$ y Edna Luna(2) \\ (1) Universidad Xochicalco. Dirección: San Francisco \#1139 Fracc. Misión, Ensenada, B.C.- \\ México. (e-mail: cecyosuna@gmail.com; cecyosuna@xochicalco.edu.mx) \\ (2) IIDE-UABC, México. Dirección: Km 106 carretera Tijuana-Ensenada, Ensenada, B.C.-México. \\ (e-mail: eluna@uabc.edu.mx)
}

Recibido May. 16, 2011; Aceptado Jun. 13, 2011; Versión final recibida Jun. 27, 2011

\begin{abstract}
Resumen
Se presenta un estudio que identifica los valores éticos que los docentes de las áreas de Ciencias Naturales e Ingeniería y Tecnología de la Universidad Autónoma de Baja California, México recomiendan fomentar en la formación universitaria. Se realizó un estudio de caso bajo un enfoque cualitativo y con un análisis de contenido se identificaron los valores de honestidad, responsabilidad y respeto. Los docentes sugirieron diversas estrategias metodológicas para la promoción de dichos valores. Se encontraron similitudes entre ambas áreas de conocimiento en valores genéricos y se detectaron particularidades en valores específicos para cada profesión. Se concluye que estos resultados podrían ser útiles para orientar las acciones educativas de la Universidad, a fin de formar el capital humano capaz de desempeñarse en su vida profesional con un claro compromiso de responsabilidad social.
\end{abstract}

Palabras clave: valores profesionales, honestidad, responsabilidad, respeto, ética

\section{Ethical Values in the University Education in the Areas of Natural Sciences and Engineering and Technology, in the Context of the Knowledge Society}

\begin{abstract}
This paper presents a study that identifies the ethical values that the professors of the academic area of Natural Sciences and Engineering and Technology of the Universidad Autonoma of Baja California, in México, recommend promoting in teaching and education. A study case was analyzed using a qualitative approach and with the technique of content analysis values such as honesty, responsibility and respect were identified. Professors suggested various methodological strategies for the promotion of those values. There were similarities between both areas of knowledge in generic values, but differences were found in some specific values. It is concluded that these results could be useful to guide the educational activities of the University, to build the human capital capable of developing their professional life with clear commitment of social responsibility.
\end{abstract}

Keywords: professional values, honesty, responsibility, respect, ethics 


\section{INTRODUCCIÓN}

A nivel mundial se habla de una tercera revolución industrial; término que refiere al amplio desarrollo de las nuevas tecnologías. Esta revolución ha propiciado adelantos científicos y técnicos que han originado una mayor generación de conocimiento; a tal punto que la sociedad de hoy se califica como sociedad del conocimiento, pues privilegia a éste como impulsor del desarrollo (UNESCO, 2005).

Las políticas internacionales impulsadas por la OCDE, la UNESCO, el Fondo Monetario Internacional y el Banco Mundial, usan el término sociedad del conocimiento en sus referentes internacionales e indican que uno de los objetivos de la sociedad del conocimiento es promover un proyecto social que vincule relaciones entre los países, orientado a la internacionalización de la educación superior así como a la articulación de procesos productivos y de mercado, distribuidos en diferentes regiones del mundo y enlazados por la comunicación digital (UNESCO, 2007, Orozco, 2009). En este contexto, las sociedades deberán compartir el conocimiento a fin de que su desarrollo sea acorde con el del ser humano y de la vida, a la par que evolucionan los desarrollos tecnológicos.

Bajo esta óptica, el desarrollo del conocimiento y su transferencia afecta no sólo el modo de operación de las economías mundiales, sino también impacta a los sistemas de educación superior. Lo anterior ha provocado que las instituciones educativas se enfrenten a nuevos desafíos, puesto que su papel es fundamental en la creación de la nueva infraestructura intelectual que será responsable de la producción, utilización y aplicación del saber (Banco Mundial, citado en Godinas, 2001). Las Instituciones de Educación Superior (IES) deben formar el capital humano en competencias profesionales y técnicas, así como en valores congruentes con el actual mercado laboral, mismo que se encuentra en constante transformación en un entorno globalizado (Banco Mundial, citado en Godinas, 2001; UNESCO, 2005). Así, mediante la formación profesional, es de esperarse que los universitarios desarrollen competencias dirigidas a su empleabilidad (Casares, Carmona y Martínez, 2010).

Este marco de referencia pone de manifiesto la necesidad de sentar bases éticas que orienten a las sociedades del conocimiento en su evolución; sustentadas en una ética de responsabilidad y libertad, basada en el aprovechamiento compartido de los conocimientos (Credé y Mansell, 1998). Se afirma que las competencias profesionales y éticas que las IES promuevan en sus estudiantes, constituirán el capital social necesario para construir sociedades civiles más prósperas unidas desde una perspectiva social (Banco Mundial, citado en Godinas, 2001). Por lo que hoy mas que nunca, es importante que el sistema de educación superior enfatice la formación valoral orientada al desarrollo de competencias profesionales y sociales (UNESCO, 1998). La UNESCO ya desde la década pasada sugirió que para promover la responsabilidad social, los programas de estudio deben fortalecer los enfoques humanistas y fomentar en los estudiantes valores como el compromiso social; promoviendo el interés de contribuir con responsabilidad en la solución de los problemas más urgentes de la humanidad (De Allende, Díaz y Gallardo, 1998; UNESCO, 1998).

Es evidente que existe una vinculación fuerte entre las competencias profesionales y los valores, mismos que no pueden ser soslayados de la formación universitaria. De acuerdo a Casares, Carmona y Martínez (2010), los valores dan fundamento a las competencias, pues si el valor esta ausente, "entonces la competencia será inadecuada en el contexto, indeseada e indeseable" (p.6). Los autores concluyen que la formación universitaria en la actualidad debe comprometerse con el desarrollo moral de los universitarios de manera contundente e intencional.

Bajo el panorama de la sociedad del conocimiento, cada vez se depende más de la ciencia y la tecnología, dado que los procesos de producción, los alimentos, las medicinas, la educación, la comunicación, etc., son campos que están ligados al desarrollo tecnológico-científico. Las dos disciplinas son sinérgicas, ambas necesitan plantear modelos, una para generar el conocimiento y la otra para resolver problemas. A decir de Tarazona (2003), el desarrollo tecnológico depende estrechamente de la ciencia básica y el progreso de la ciencia depende también de la tecnología. Así, en la sociedad del conocimiento actual la frontera entre ellas es tenue y esa relación tan estrecha ha ido evolucionando a una nueva modalidad de ciencia llamada tecnociencia. Esa 
frontera tenue se hace visible cuando las redes entre la ciencia y la tecnología se han consolidado. La tecnociencia no solo pretende explicar el mundo sino transformarlo, pero nunca al margen de los valores, pues es imperativo considerar las consecuencias y efectos de cualquier aplicación tecnocientífica (Tarazona, 2003). En este contexto la demanda de científicos e ingenieros se ha incrementado por lo cual, la pertinencia de la formación de perfiles profesionales orientados a las ciencias e ingenierías es innegable.

Sin embargo, a pesar de la relevancia del tema no hay señalamientos claros en la literatura sobre qué valores se deben privilegiar en la enseñanza de los perfiles profesionales de las áreas de ciencias e ingeniería. De manera general se indica que las profesiones no sólo son portadoras del conocimiento científico, sino que los profesionistas son personas que deben hacer una contribución benéfica a la sociedad procurando mejorar la calidad de vida (Cortina, 2002). En el mismo orden de ideas, Hortal (2010) coincide al enfatizar la importancia del sentido social como componente inherente a la profesión. Como se aprecia, las profesiones no se pueden separar de la sociedad ya que están íntimamente ligadas a ella (Cortina, 2002).

Tampoco hay lineamientos definidos sobre con base en qué estrategias metodológicas se deben promover los valores. Hirsch (2004) indica que se deben promover por medio de la inserción de asignaturas de ética profesional en los planes de estudios; Schmelkes (2001) opina que es a través de la reflexión y autonomía personal; es decir, cada individuo debe construir libremente su esquema valoral. Otros autores mencionan que debe ser por medio de la discusión de dilemas morales (Ortega et al., 1996), por el estudio de casos (Ruiz, 1994), u orientarse al desarrollo de competencias, actitudes y conductas que supongan un alto nivel de autocontrol en el alumno (autorregulación de la conducta) (Ortega et al., 1996). Sin embargo, algunos estudios empíricos hacen evidentes las limitaciones en las estrategias que fomenten la formación integral de los universitarios (Luna, Valle y Osuna 2010), así como los valores específicos que se deben fomentar en cada una de las titulaciones universitarias (Osuna y Luna, 2008).

Con base en el análisis hasta aquí expuesto, el objetivo de este estudio fue identificar en opinión de los docentes qué valores ético profesionales se deben fomentar en la formación universitaria de las áreas de ciencias naturales e ingeniería y tecnología; así como las estrategias metodológicas que recomiendan para la formación de las competencias éticas necesarias para dichas áreas de conocimiento.

\section{METODOLOGIA}

El trabajo de campo tuvo como escenario la Universidad Autónoma de Baja California (UABC), Campus Ensenada, México. De manera específica se trabajó con docentes de las licenciaturas de las áreas de Ciencias Naturales (Biología y Oceanología) e Ingeniería y Tecnología (Ingeniería Civil, Ingeniería Electrónica, Ingeniería en Computación, Ingeniería Industrial y Ciencias Computacionales).

\section{Diseño de la investigación}

Esta investigación se diseñó como un estudio de caso en el que se consideró importante recabar la opinión de los docentes acerca de la formación en valores éticos en las áreas de conocimiento mencionadas. Los propósitos específicos fueron: a) registrar los valores ético profesionales que los docentes sugieren privilegiar en la enseñanza universitaria, b) identificar las estrategias de enseñanza que ellos utilizan para la promoción de dichos valores y, c) los temas que sugieren incluir en los programas de las asignaturas. No se buscó la generalización de los resultados en un sentido cuantitativo, puesto que los resultados de un estudio de caso expresan solo los aspectos singulares de la situación estudiada (Yin, 2003).

Participantes

Se determinó una muestra intencional de 31 profesores de las áreas de Ciencias Naturales e Ingeniería y Tecnología. Las sub-muestras se seleccionaron bajo dos criterios: a) Docentes que imparten el curso de Ética Profesional y asignaturas interdisciplinarias en las que se fomentan valores $\mathrm{y}, \mathrm{b})$ docentes que imparten diversas asignaturas en las tres etapas curriculares que 
conforman los planes de estudio (etapa básica de primer a tercer semestre, etapa disciplinaria de cuarto a sexto y etapa terminal de séptimo a noveno semestre de la carrera). Bajo el primer criterio aceptaron participar 3 profesores y bajo el segundo participaron 28 profesores.

El $68 \%$ de la muestra fueron varones y $32 \%$ mujeres, $58 \%$ imparte clases en el área de Ingeniería y Tecnología y $42 \%$ en Ciencias Naturales. El $54 \%$ de los docentes tiene maestría, 30\% doctorado, $13 \%$ licenciatura y $3 \%$ alguna especialidad. En relación a la etapa curricular en que dan clases $22 \%$ enseña en la etapa básica, $42 \%$ en la disciplinar y $36 \%$ en la etapa terminal.

\section{Instrumento}

Se diseñó un cuestionario con cuatro preguntas abiertas: ¿cuáles son los valores de ética profesional que usted considera se deben promover en la enseñanza de esta profesión?, ¿cómo sugiere que los valores de ética profesional sean enseñados?, ¿qué estrategias metodológicas utiliza en su práctica docente para fomentar los valores de ética profesional? y, ¿qué temas o contenidos considera que deben ser enseñados?

\section{Procedimiento}

Las respuestas completas de los docentes se analizaron por medio de la técnica de análisis de contenido, considerando para ello categorías y unidades de análisis en el texto (Titscher, Meyer, Wodak y Vetter, 2000). El procedimiento se dividió en dos momentos:

1. Definición de las categorías de análisis previas: estas se derivaron de los planteamientos teóricos de la literatura internacional y nacional acerca de los valores que se deben fomentar en la formación ético profesional. Asimismo, se consideró la posibilidad de identificar otras categorías que emergieran de las respuestas de los docentes.

2. Análisis de contenido: a) En las respuestas completas de los docentes se ubicaron las secciones que mencionaran los valores éticos recomendados, las estrategias para su enseñanza y los contenidos que deben ser enseñados.

b) Se identificaron como unidades de análisis las oraciones mencionadas por los docentes que hacían referencia a los valores éticos, así como las estrategias metodológicas y los contenidos recomendados para su enseñanza.

c) Se identificó la presencia o ausencia de las categorías previas en las unidades de análisis, así como las nuevas categorías que emergieron del análisis.

d) De acuerdo con la frecuencia de aparición de las categorías, se identificaron los valores presentes y los ausentes.

e) Por último, se jerarquizaron los valores éticos, las estrategias y los contenidos que los docentes recomendaron privilegiar en la formación universitaria y se interpretaron los hallazgos.

\section{RESULTADOS}

Dado la gran diversidad de categorías identificadas, se optó por seleccionar todas aquellas que obtuvieron una frecuencia (f) de aparición mínima de tres o más ocurrencias. Los resultados se presentan de acuerdo al orden de las preguntas.

En el área de ciencias naturales los docentes consideraron en orden de importancia que se debe promover honestidad, responsabilidad, el cuidado del ambiente, el compromiso social y el respeto. Los docentes de las ingenierías consideran que se debe promover honestidad, respeto, responsabilidad, puntualidad, compromiso social, congruencia, confianza y justicia.

Respecto a cómo enseñar los valores, los docentes de ciencias naturales sugieren que sean enseñados a través de una asignatura (un curso específico sobre ética profesional). En segundo 
lugar por medio del ejemplo (es decir que los profesores vivan los valores). En términos de la cotidianeidad, refieren a vivirlos como parte de la rutina diaria y aún afuera del recinto universitario. Como temas transversales, indicaron que se deben incorporar en los objetivos de todas las asignaturas. Por último, la reflexión que se orienta a recapacitar permanentemente en el trabajo que se desempeña y los valores implicados en ello.

Los docentes de ingeniería consideraron en orden de importancia: el ejemplo de docentes y directivos observando su conducta y desarrollando sus labores intachablemente. Como estrategias metodológicas recomendaron usar de videos, anécdotas, lecturas y ejercicios relacionados con los valores, la cotidianeidad referida a que los valores se vivan a partir de la práctica cotidiana, el estudio de casos que hace mención a ejemplificar y analizar casos reales y por último, una asignatura.

En relación a las estrategias metodológicas, las más utilizadas en el área de ciencias naturales son: estudio de casos, ejemplo, cotidianeidad, reflexión y, diálogo. Por parte de los docentes de las ingenierías: el ejemplo, cotidianeidad, estudio de casos, diálogo y trabajo grupal. El ejemplo, la cotidianeidad y el estudio de casos están definidos líneas arriba, respecto al diálogo, lo relacionan a pláticas con los alumnos sobre los valores y sobre su comportamiento. Por último, el trabajo grupal es utilizado en actividades colaborativas dentro del aula.

Respecto a los temas específicos que se deben enseñar, según los docentes de ciencias naturales los valores no pueden ser enseñados; es decir, no se aprenden enseñando contenidos o teoría. Los docentes de las ingenierías indicaron que se debe enseñar la honestidad apegada a la actuación profesional basada en la integridad, es decir, no efectuar ilícitos como plagio o realizar piratería. La categoría valores se mencionó de manera genérica sin especificarse qué valores deben ser enseñados. El Compromiso social lo orientaron a la responsabilidad social, considerando el impacto que se tiene en la sociedad con la actuación profesional y, la moral, fue referida a las costumbres, conducta humana y valores morales

Los resultados encontrados de acuerdo a cada pregunta y por área de conocimiento se muestran en la Tabla 1.

Tabla1. Resultados del análisis de contenido a las respuestas de los docentes de las áreas de Ciencias Naturales e Ingeniería y Tecnología.

\begin{tabular}{|c|c|c|}
\hline Pregunta & $\begin{array}{c}\text { Docentes área de } \\
\text { Ciencias Naturales }\end{array}$ & $\begin{array}{c}\text { Docentes área de } \\
\text { Ingeniería y Tecnología }\end{array}$ \\
\hline $\begin{array}{l}\text { ¿Cuáles son los valores ético } \\
\text { profesionales que usted considera } \\
\text { se deben promover en el } \\
\text { enseñanza de esta profesión? }\end{array}$ & $\begin{array}{ll}\text { 1. } & \text { Honestidad }(\mathrm{f}=10) \\
\text { 2. } & \text { Responsabilidad }(\mathrm{f}=8) \\
\text { 3. } & \text { Cuidado del ambiente } \\
& (\mathrm{f}=6) \\
\text { 4. } & \text { Compromiso social }(\mathrm{f}=4) \\
\text { 5. } & \text { Respeto }(\mathrm{f}=3)\end{array}$ & $\begin{array}{ll}\text { 1. } & \text { Honestidad }(\mathrm{f}=17) \\
\text { 2. } & \text { Respeto }(\mathrm{f}=9) \\
\text { 3. } & \text { Responsabilidad }(\mathrm{f}=8) \\
\text { 4. } & \text { Puntualidad }(\mathrm{f}=6) \\
\text { 5. } & \text { Compromiso social, } \\
\text { 6. } & \text { congruencia y confianza }(\mathrm{f}=4)\end{array}$ \\
\hline $\begin{array}{l}\text { ¿Cómo sugiere que los valores de } \\
\text { ética profesional sean enseñados? }\end{array}$ & $\begin{array}{ll}\text { 1. } & \text { Asignatura }(\mathrm{f}=8) \\
\text { 2. } & \text { El ejemplo }(\mathrm{f}=7) \\
\text { 3. } & \text { Cotidianeidad }(\mathrm{f}=6) \\
\text { 4. } & \text { Temas transversales }(\mathrm{f}=4) \\
\text { 5. } & \text { Reflexión }(\mathrm{f}=3)\end{array}$ & $\begin{array}{ll}\text { 1. } & \text { El ejemplo }(\mathrm{f}=12) \\
\text { 2. } & \text { Herramientas didácticas }(\mathrm{f}=6) \\
\text { 3. } & \text { Cotidianeidad }(\mathrm{f}=5) \\
\text { 4. } & \text { Estudio de casos }(\mathrm{f}=4) \\
\text { 5. } & \text { Asignatura }(\mathrm{f}=3)\end{array}$ \\
\hline $\begin{array}{l}\text { ¿Qué estrategias metodológicas } \\
\text { utiliza en su práctica docente para } \\
\text { fomentar los valores ético } \\
\text { profesionales? }\end{array}$ & $\begin{array}{ll}\text { 1. } & \text { Estudio de casos }(\mathrm{f}=7) \\
\text { 2. } & \text { El ejemplo }(\mathrm{f}=4) \\
\text { 3. } & \text { Cotidianeidad, reflexión y } \\
\text { diálogo }(\mathrm{f}=3)\end{array}$ & $\begin{array}{ll}\text { 1. } & \text { El ejemplo }(\mathrm{f}=10) \\
\text { 2. } & \text { Cotidianeidad }(\mathrm{f}=8) \\
\text { 3. } & \text { Estudio de casos }(\mathrm{f}=4) \\
\text { 4. } & \text { Diálogo y trabajo grupal }(\mathrm{f}=3)\end{array}$ \\
\hline $\begin{array}{l}\text { ¿Qué temas o contenidos } \\
\text { considera que deben ser } \\
\text { enseñados? }\end{array}$ & $\begin{array}{l}\text { 1. No pueden enseñarse los } \\
\text { valores }(f=4) \\
\text { 2. Ética profesional }(f=3)\end{array}$ & $\begin{array}{ll}\text { 1. } & \text { Honestidad }(f=5) \\
\text { 2. } & \text { Valores }(f=4) \\
\text { 3. } & \text { Compromiso social y moral } \\
(\mathfrak{f}=3)\end{array}$ \\
\hline
\end{tabular}




\section{CONCLUSIONES}

a) Se aprecian similitudes y diferencias entre las opiniones de los docentes de ambas áreas de conocimiento. Respecto a las similitudes, ambos grupos mencionan que se debe privilegiar la honestidad en la formación ético profesional. Otras coincidencias se detectan al sugerir la responsabilidad, el respeto y el compromiso social. Entre las discrepancias, los docentes del área de ciencias naturales ponderan en tercer lugar el cuidado del ambiente, valor sumamente relacionado con el ejercicio de las profesiones de ésa área; mientras que en las ingenierías no obtuvo ninguna mención. Por su parte, los docentes de ingeniería y tecnología consideraron la puntualidad, congruencia, confianza y justicia como valores importantes en la formación de los ingenieros. Cabe destacar que el compromiso social es un valor que se recomienda ampliamente en la literatura para la formación profesional de todas las titulaciones (De Allende, Díaz y Gallardo, 1998; UNESCO, 1998, Cortina, 2002 y Hortal, 2010). Por el contrario de valores como puntualidad, congruencia y confianza, que son poco mencionados.

b) Hay coincidencia aunque en diferente orden, en tres de las sugerencias para la enseñanza de los valores éticos: una asignatura que en el área de ciencias naturales fue considerada como la principal sugerencia, pero para las ingenierías fue la última; el ejemplo: en el área de ciencias naturales fue ubicado en segundo sitio mientras que en ingeniería fue la categoría más importante y cotidianeidad: misma que en ambos casos fue jerarquizada en tercer lugar.

c) Respecto a las estrategias metodológicas que usan los docentes las convergencias son: el ejemplo que en ciencias naturales fue la segunda estrategia utilizada en su práctica docente para fomentar los valores éticos; mientras que para los docentes de las ingenierías es la estrategia número uno. El estudio de caso en opinión de los docentes de ciencias naturales fue la estrategia número uno, misma que ocupa tercer lugar en opinión de los maestros de las ingenierías. Cotidianeidad, de acuerdo a los docentes de ciencias naturales ocupa el tercer lugar de importancia, mientras que en el área de ingeniería y tecnología se considera la segunda estrategia más utilizada y el diálogo, en ambas áreas se ponderó en cuarto lugar.

d) En la última respuesta no hubo coincidencia, en el área de ciencias naturales se opinó que los valores éticos no pueden ser enseñados, mientras que en ingeniería y tecnología se privilegia la honestidad, valores en general, compromiso social y la moral, como temas o contenidos a enseñar.

e) Los resultados indicaron que se privilegian valores que son genéricos para cualquier profesión, pero también refieren valores específicos; en ciencias naturales señalaron el cuidado del ambiente en lugar relevante, lo que podría implicar que la formación ética de los profesionales de esta área deben considerar el desarrollo sostenido de la sociedad en armonía con el cuidado del medio ambiente. En ingeniería y tecnología es evidente que los docentes tienen clara la importancia de valores específicos para la formación de un ingeniero por ejemplo: respeto, puntualidad, congruencia y confianza; mismos que no fueron referidos por los docentes del área de las ciencias.

f) Es claro que existe consenso entre los profesores en la necesidad de promover valores en la formación universitaria, sin embargo, no hay acuerdo sobre cuáles valores específicos se deben privilegiar, la gran diversidad de menciones da cuenta de ello. Con lo anterior, se confirma la opinión de Zárate (2001) quien indica que en el ámbito académico no hay conformidad sobre cuáles valores profesionales deben promoverse.

g) Destacan categorías que se relacionan con el currículum oculto: el ejemplo y la cotidianeidad; sin embargo, el problema con el curriculum oculto es que considera elementos sobre los cuales ni los docentes ni las instituciones educativas tienen una intencionalidad explícita (Díaz, 2006). Lo anterior refleja que la formación ética pudiera estar siendo abordada dentro del aula a criterio de los docentes. 
h) Surge la necesidad de estudiar ampliamente cuáles son los recursos didácticos más adecuados para la formación de valores éticos. A decir de López (2007) los profesores son los principales actores en el proceso de formación de los futuros profesionistas, pues contribuyen a crear ambientes de aprendizaje y relaciones educativas que darán sustento a la formación profesional.

i) En el contexto de la sociedad del conocimiento caracterizado por el amplio desarrollo de la ciencia y la tecnología y la evolución hacia la tecnociencia, habría que analizar si las recomendaciones de los docentes se ajustan a una formación ética sólida, pues la sociedad de conocimiento tiene como contraparte ciertos riesgos dado que los productos de las actividades científico-tecnológicas mal utilizados, podrian tener consecuencias serias y dañar las condiciones de vida de la sociedad.

j) Por último, identificar los valores que los docentes consideran imprescindibles en la formación de los profesionales de las ciencias e ingenierías y las estrategias que en su opinión son las mas adecuadas para promoverlos, podría orientar las acciones educativas de las IES, a fin de responder con la formación del capital humano capaz de desempeñarse adecuadamente en el entorno social actual, con un compromiso de responsabilidad social ante la creación, transferencia y aplicación del conocimiento.

\section{REFERENCIAS}

Casares, P., Carmona, G. y Martínez, F. Valores profesionales en la formación universitaria. Revista Electrónica de Investigación Educativa. Consultado el 14 de Abril de 2011 en:

http://redie.uabc.mx/contenido/NumEsp2/contenido-casares.html (2010).

Cortina, A. Ética y formación universitaria. Revista Iberoamericana de Educación. 28. Consultado el 10 de Junio de 2011 en: http://www.campus-org/revista/rie28a01.htm (2002).

Crede, A. y Mansell, R. Knowledge societies. in a nutshell: Information technologies for sustainable development. Ottawa, Canadá: IDRC (1998).

De Allende, M., Díaz, G., y Gallardo, C. La Educación Superior en México y en los Países en vías de desarrollo desde la óptica de los Organismos Internacionales. México: Libros en línea ANUIES consultado el 5 de Mayo de 2011 en:

http://www.anuies.mx/servicios/p_anuies/publicaciones/libros/lib30/0.htm (1998).

Díaz, A. La educación en valores: avatares del currículo formal, oculto y los temas transversales. Revista Electrónica de Investigación Educativa. 8 (1). Consultado el 6 de Abril de 2011 en: http:// redie.uabc.mx/vol8no1/contenido-diazbarriga2.html (2006).

Godinas, L. Construcción de sociedades de conocimiento: nuevos retos para la educación superior. Resumen ejecutivo extraído del reporte del Banco Mundial. Perfiles Educativos. 23 (092), 98-113 (2001).

Hirsch, A. Utopía y Universidad. La enseñanza de la ética profesional. Reencuentro. Análisis de Problemas Universitarios. 41, 31-37 (2004).

Hortal, A. Ética general de las profesiones. Espaňa: Editorial Desclée de Brouwer (2010).

López, R. Valores profesionales en la formación universitaria. La dimensión social de los valores del profesorado. Revista Reencuentro. Análisis de problemas universitarios. 49, 60-64 (2007).

Luna, E., Valle, M. y Osuna, C. Los rasgos de un "buen profesional", según la opinión de estudiantes universitarios en México. Revista Electrónica de Investigación Educativa. Consultado el día 10 de febrero de 2011 en: http://redie.uabc.mx/NumEsp2/contenido-luna3.html (2010). 
Orozco, B. Competencias y curriculum: una relación tensa y compleja. Ponencia presentada en el $X$ Congreso Mexicano de Investigación Educativa realizado en Veracruz, México. Consultado el 22 de marzo de 2011 en: http://www.riseu.unam.mx/documentos/acervo_documental/txtid0057.pdf (2009)

Ortega, P., Mínguez, R. y Gil, R. Valores y educación. España: Editorial Ariel (1996).

Osuna, C. y Luna E. Los Valores Ético-profesionales que Promueven los Documentos Rectores de una Universidad Pública en México. Archivos Analíticos de Políticas Educativas. 16 (15). Consultado el 14 de Abril de 2011 en: http://epaa.asu.edu/epaa/v16n15/ (2008).

Ruíz, M., La enseñanza de los valores. Utopías. 37-43 (1994).

Schmelkes, S., La formación en valores. Revista Intercontinental de Psicología y Educación. 3 (2), 53-57 (2001).

Tarazona, L., Tecnociencia, sociedad y valores. Revista Ingeniería y Desarrollo. 14, 38-59.

Consultado el 20 de Junio de 2011 en:

http://ciruelo.uninorte.edu.co/pdf/ingenieria_desarrollo/14/tecnociencia_sociedad_y_valores.pdf (2003).

Titscher, S., Meyer, M., Wodak, R. y Vetter, E. Methods of Text and Discourse Analysis. Londres: Sage (2000).

UNESCO. Declaración Mundial sobre la Educación Superior en el siglo XXI: Visión y Acción.

Consultado el 10 de Septiembre de 2010 en:

http://www.unesco.org/education/educprog/wche/declaration_spa.htm (1998).

UNESCO. Hacia las Sociedades del Conocimiento. Ediciones UNESCO, París (2005).

UNESCO. La universidad en las sociedades del conocimiento, UNESCO, México (2007).

Yin, K. Applied Social Researching Series. Case Study Research, Design and Methods. 5, 70-92 (2003).

Zárate, R. La formación de valores y actitudes en la educación superior. En A. Hirsch (coord.). Educación y valores. Tomo II, México: Gernika (2001). 